\section{Biodiversität erfolgreich managen}

\author{
Biodiversitätsmanagement ist ein bisher wenig beachteter \\ Teilbereich des unternehmerischen Nachhaltigkeitsmanage- \\ ments. Dadurch gehen dem Biodiversitätserhalt eine zentrale \\ Akteursgruppe und den Unternehmen Erfolgspotenziale \\ verloren. Von Stefan Schaltegger
}

Gestaltung von Leistungserstellungsprozessen, Produkten und Projekten, um eine nachhaltige Entwicklung von Unternehmen und Gesellschaft durch die Erhaltung der biologischen Vielfalt zu erreichen. Dieser Ansatz unterscheidet sich durch das Ziel einer unternehmerischen Mehrwertgenerierung von philanthropischen Projekten, die für ihre Umsetzung auf einen (möglicherweise unnachhaltig geschaffenen) wirtschaftlichen Erfolg des Unternehmens angewiesen sind.

\section{Handlungsfelder des Biodiversitätsmanagements}

W ir erleben einen drastischen weltweiten Rückgang an Biodiversität. So sank der vom World Wide Fund For Nature (WWF) veröffentlichte Global Living Planet Index zu Biodiversität seit 1970 um 28 Prozentpunkte (WWF 2012). Dabei wird der Erhalt der Artenvielfalt vor allem als eine Aufgabe des Staates und von Naturschutzorganisationen wahrgenommen. In Unternehmen erhält das Thema derzeit noch wenig Beachtung. Umfragen des Corporate Sustainability Barometers zeigen für die letzten Jahre, dass Biodiversität bei den größten Unternehmen Deutschlands die geringste Priorität unter allen Nachhaltigkeitsthemen aufweist (Schaltegger et al. 2012). Dies ist sehr bedauerlich, da einerseits wesentliche Problemlösungsbeiträge von Unternehmen brach liegen und andererseits der Beitrag eines systematischen Biodiversitätsmanagements zur Reduzierung unternehmerischer Risiken und Realisierung von Chancen meist übersehen wird.

Dennoch scheint Artenvielfalt als Unternehmensthema erkannt worden $\mathrm{zu}$ sein. So hat die Bundesregierung mit der Gründung der „Biodiversity in Good Company Initiative“ ein Unternehmensforum geschaffen, das sich der praxisorientierten Entwicklung des unternehmerischen Biodiversitätsmanagements widmet und den Erfahrungsaustausch zwischen engagierten Unternehmen fördert. In diesem Rahmen wurde ein Handbuch zum unternehmerischen Biodiversitätsmanagement entwickelt (Schaltegger/ Beständig 2010), dessen weitere Konkre- tisierung und Umsetzung mit Unterstützung des Bundesumweltministeriums (BMU) und des Bundesamts für Naturschutz (BfN) zu einer Reihe von branchen- und themenspezifischen Leitfäden geführt hat (z.B. für Finanzinstitutionen Beständig et al. 2011, zu Liegenschaftsverwaltung, Einkauf und Marketing Beständig / Wuczkowski 2012). Aus den dokumentierten Fallstudien wird auch deutlich, dass eine steigende Anzahl an Unternehmen sich des Themas annimmt.

Ziel des unternehmerischen Biodiversitätsmanagements ist, die systematische

Abbildung 1: Basiskonzept des unternehmerischen Biodiversitätsmanagements

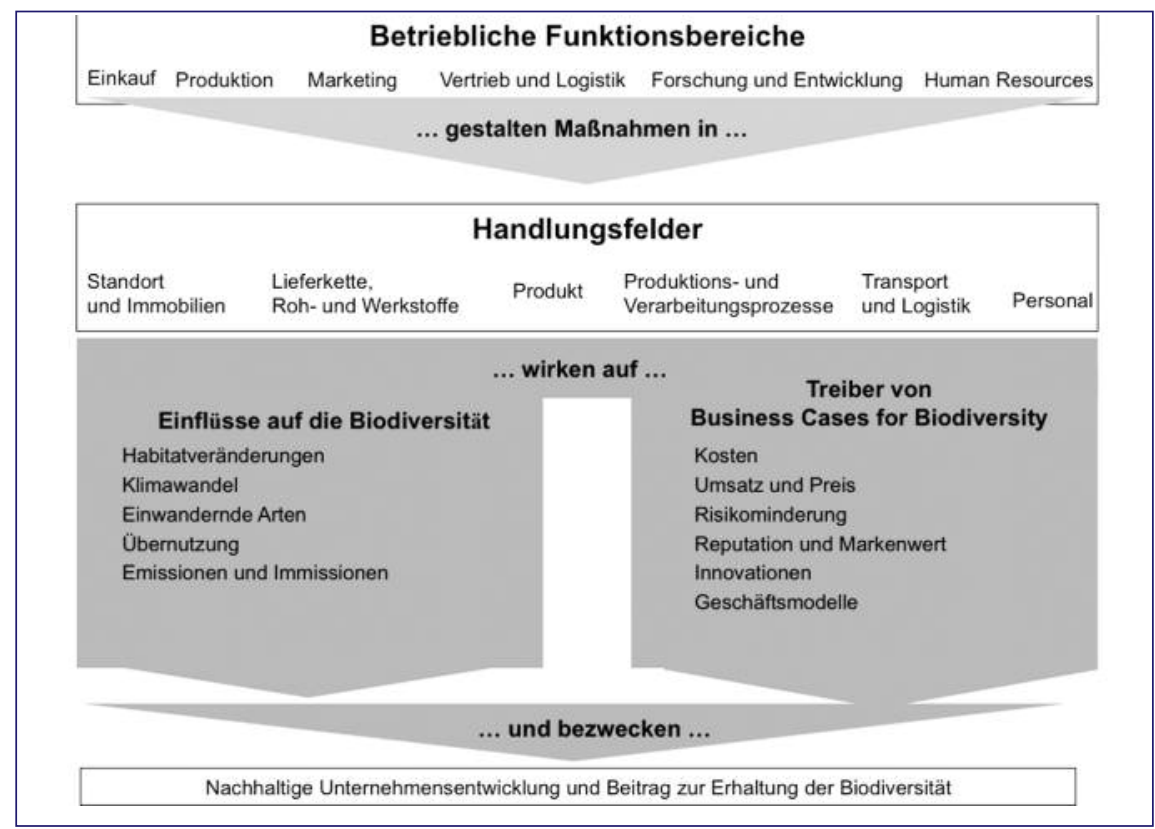

Quelle: Schaltegger / Beständig 2010
Biodiversität umfasst die genetische Vielfalt sowie die Vielfalt an Ökosystemen, Tier- und Pflanzenarten. Als wesentliche Ursachen für den Biodiversitätsverlust gelten die Veränderungen von Lebensräumen durch großflächige Kultivierungsmaßnahmen originärer Lebensräume, landwirtschaftliche Überdüngung, intensive Nutzungsformen wie Überfischung und Abholzung, invasive Arten, Emissionen und den Klimawandel (CBD 2006). Aus diesen Einflussfaktoren auf den Biodiversitätsverlust können verschiedene Handlungsfelder des unternehmerischen Biodiversitätsmanagements abgeleitet 
werden, um den Verlust an biologischer Vielfalt einzudämmen und gleichzeitig einen unternehmerischen Mehrwert zu generieren (vgl. Abbildung 1):

- Standorte und Immobilien wegen ihres Flächenbedarfs und der Gestaltungsmöglichkeiten wie Begrünung von Dächern, Schaffen von Nistplätzen usw.

- Lieferketten, da sich die Beschaffung von Roh- und Werkstoffen auf Flächen und Ökosysteme außerhalb des Eigentumskreises des Unternehmens auswirkt (zum Beispiel auf Fischbestände oder die Forstwirtschaft).

- Produkte, da je nach Produktgestaltung (beispielsweise Ausgestaltung eines Touristikangebotes) und -zusammensetzung (zum Beispiel toxische Stoffe) unterschiedliche Wirkungen auf die Biodiversität ausgehen.

- Leistungserstellungsprozesse durch unterschiedliche Emissionen.

- Transporte durch die Verbreitung gebietsfremder Arten und da Verkehrswege Lebensräume zerschneiden können.

【 Personal, da die täglichen Entscheidungen und Handlungen von Mitarbeitenden Biodiversitätswirkungen auslösen.

\section{Business Cases for Biodiversity}

Die Verbindung aus Biodiversitätserhalt und Stärkung der Wettbewerbsfähigkeit charakterisiert einen „Business Case for Biodiversity“. Ein solcher existiert weder a priori noch tritt er automatisch auf. Er ist vielmehr das Ergebnis eines gezielten Managementprozesses, der BusinessCase-Treiber systematisch anspricht. Ansätze zur Kostenreduzierung bestehen in der Extensivierung der Grundstückspflege und der Reduktion des Ressourcenverbrauchs. Biodiversitätsschutz kann Teil des Risikomanagements sein, wenn Ökosystemdienstleistungen wie die Trinkwasserversorgung gesichert, nachwachsende Rohstoffe geschützt oder Reputationsrisiken verhindert werden (WBCSD 2008). Da die Natur für viele Menschen ein wichtiges Anliegen ist, kann Biodiversitätsma- nagement die Unternehmensreputation und die Markenidentifikation steigern.

\section{Ausblick}

Unternehmen sind nicht nur Problemverursacher, sondern können auch bedeutende Problemlöser sein. Mit der Identifizierung der unternehmensrelevanten Handlungsfelder und der Analyse von Möglichkeiten, einen Business Case for Biodiversity zu kreieren, lassen sich der unternehmerische Nutzen darstellen und Argumentationslinien erarbeiten.

\section{Literatur}

CBD (Convention on Biological Diversity) (2006): Global Biodiversity Outlook 2. Genf.

Beständig, U. / Detlefsen, I. / Jahn, K. (2011): Leitfaden für den Finanzsektor zur Beurteilung von Biodiversitätsrisiken und -chancen, Augsburg, Verein für Umweltmanagement und Nachhaltigkeit in Finanzinstituten e.V. (VfU).

Beständig, U. / Wuczkowski, M. (2012): Biodiversität im unternehmerischen Nachhaltigkeitsmanagement: Chancen und Ansätze für Einkauf, Marketing und Liegenschaftsmanagement, Lüneburg, CSM.

Schaltegger, S. / Beständig, U. (2010): Handbuch unternehmerisches Biodiversitätsmanagement, Lüneburg/Eschborn/Berlin, CSM/ Business and Biodiversity Initiative der Bundesregierung/BMU.

http://www.bmu.de/naturschutz_biologische_vielfalt/downloads/doc/46143.php

Schaltegger, S./ Harms, D./ Hoerisch, J. / Windolph, S. (2012): Corporate Sustainability Barometer 2012, Lüneburg, CSM.

UNEP (United Nations Environmental Programme Finance Initiative) (2008): Biodiversity and Ecosystem Services. Bloom or Bust? Genf.

WBCSD (World Business Council for Sustainable Development), Meridian Institute \& WRI (World Resource Institute) (2008): Corporate ecosystem services review - guidelines for identifying business risks and opportunities arising from ecosystem change. Genf.

WWF (World Wide Fund For Nature) (2012) Living planet report 2012. Gland, WWF.

\section{IUTOR + KONTAKT}

Prof. Dr. Stefan Schaltegger ist Ordinarius für Nachhaltigkeitsmanagement an der Leuphana Universität Lüneburg und Leiter des Center for Sustainability Management (CSM).

Prof. Dr. Stefan Schaltegger, Scharnhorststr. 1, 21335 Lüneburg, Tel.: +4941316772180

E-Mail: schaltegger@uni.leuphana.de

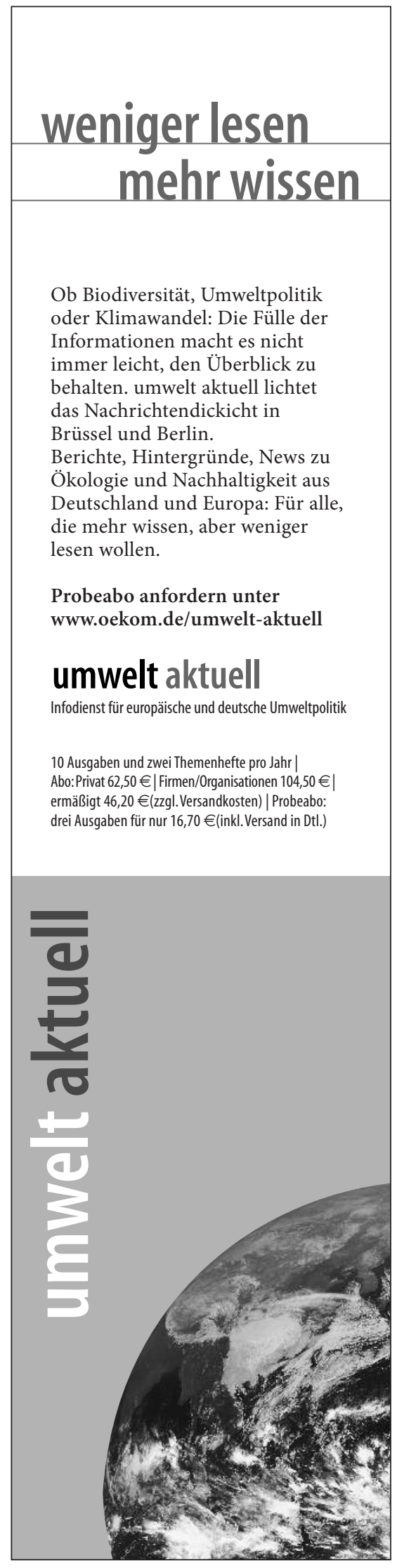


Copyright (C) 2012, IÖW und oekom Verlag. Die Nutzung des Artikels ist Abonnenten von Ökologisches Wirtschaften vorbehalten. Nachdruck und Vervielfältigung des Artikels einschließlich Speicherung und Nutzung auf optischen und elektronischen Datenträgern nur mit Zustimmung der Redaktion von Ökologisches Wirtschaften (http://www.oekologischeswirtschaften.de). 